\title{
Completion Pancreaticoduodenectomy for Hereditary Pancreatitis After Prior Puestow Procedure: A Case Report
}

\author{
James R. Nellen, Adam G. Strickland, and Charles J. Yeo
}

\begin{abstract}
Background: Hereditary pancreatitis (HP) is an uncommon condition resulting from an imbalance of pancreatic proteases. Most commonly, protease serine 1 genetic mutations are causative for HP and often result in recurrent early onset episodes of acute pancreatitis typically progressing to chronic pancreatitis, with a high risk of pancreatic cancer.

Case Presentation: A 46-year-old female with HP, confirmed by genetic testing, presented with a 7-month history of recurrent pancreatitis. She had previously undergone a distal pancreatectomy and Puestow procedure in 1992 at 21 years of age, after having pancreatitis as a teenager. The patient now had a completion pancreaticoduodenectomy and celiac ethanol nerve block.

Conclusion: A completion pancreatectomy in patients with HP can be performed after previous pancreatic surgical intervention to treat disease manifestations and as a prophylaxis against an increased risk of pancreatic adenocarcinoma.
\end{abstract}

Keywords: completion pancreatectomy; ethanol nerve block; hereditary pancreatitis; pancreaticoduodenectomy; Puestow

\section{Introduction}

Hereditary pancreatitis (HP) is an uncommon condition leading to recurrent episodes of acute pancreatitis frequently resulting in a clinical picture of chronic pancreatitis. Often initially misdiagnosed as idiopathic pancreatitis, HP presents earlier than typical acute pancreatitis caused by alcohol or gallstones, and also carries an increased risk of pancreatic adenocarcinoma. ${ }^{1-3}$ Clinical presentation results from a pathological activation of cationic trypsinogen in the pancreatic parenchyma and genetic mutation in protease serine 1 (PRSS1), serine protease inhibitor Kazal type 1 (SPINK1), cystic fibrosis transmembrane conductance receptor (CFTR), and chymotrypsin C (CTRC) are commonly present. ${ }^{3-5}$ Many HP patients with acute pancreatitis will progress to chronic pancreatitis. ${ }^{3,6}$ Although a diagnosis of HP may be made clinically, advances in genetic testing have identified numerous PRSS1 genetic mutations resulting in HP. ${ }^{3,7}$ In this report, we present a case of HP successfully managed with a completion pancreatectomy after previous pancreatic surgical intervention.

\section{Case Presentation}

A 46-year-old Caucasian female presented in November of 2017 with a longstanding history of pancreatitis. The patient is part of a family with multiple diagnosed cases of pancreatitis who had undergone genetic testing to reveal a PRSS1 gene mutation (N29I) and diseasemodifying CFTR mutation ((TG)11-5T). The patient first showed symptoms of pancreatitis when she was 13 years old. Eight years later in 1992, the patient underwent a distal pancreatectomy, splenectomy, and

Department of Surgery, Jefferson Pancreas, Biliary and Related Cancer Center, Thomas Jefferson University, Philadelphia, Pennsylvania.

*Address correspondence to: James R. Nellen, Department of Surgery, Jefferson Pancreas, Biliary and Related Cancer Center, Thomas Jefferson University, 1015 Walnut Street, Curtis Building, Suite 620, Philadelphia, PA 19107-5084, E-mail: james.nellen@jefferson.edu

(C) James R. Nellen et al. 2018; Published by Mary Ann Liebert, Inc. This Open Access article is distributed under the terms of the Creative Commons License (http://creativecommons.org/licenses/by/4.0), which permits unrestricted use, distribution, and reproduction in any medium, provided the original work is properly cited. 
cholecystectomy with modified Puestow procedure for symptoms of radiating epigastric pain, nausea, and vomiting typically lasting for 1 week and occurring three to four times per year (Fig. 1). Since that time, the patient had been followed every 6 months with accepted routine pancreatic cancer surveillance through magnetic resonance imaging and endoscopic ultrasonography. ${ }^{8}$ The patient did well until May of 2017 when she began experiencing recurring symptoms of acute pancreatitis, including dull waxing and waning epigastric pain with foods and liquids. On computed tomography scan, significant calcification in the right-sided pancreatic remnant was present (Fig. 2). Both CA 19-9 and carcinoembryonic antigen (CEA) values were elevated at 54 and 5.0, respectively. The patient elected to proceed with a completion pancreaticoduodenectomy and ethanol nerve block. Intraoperatively, there were significant adhesions present, which were lysed. The Puestow was taken down by transecting the Roux limb using a gastrointestinal anastomosis (GIA) stapler. The duodenum just distal to the pylorus was also transected using a GIA stapler in the same manner that is done during a pylorus-preserving pancreaticoduodenectomy. The hepaticojejunostomy was planned using the Roux limb from the prior Puestow procedure. The duodenojejunostomy was made with the proximal jejunum just distal to the ligament of Trietz (Fig. 3). The

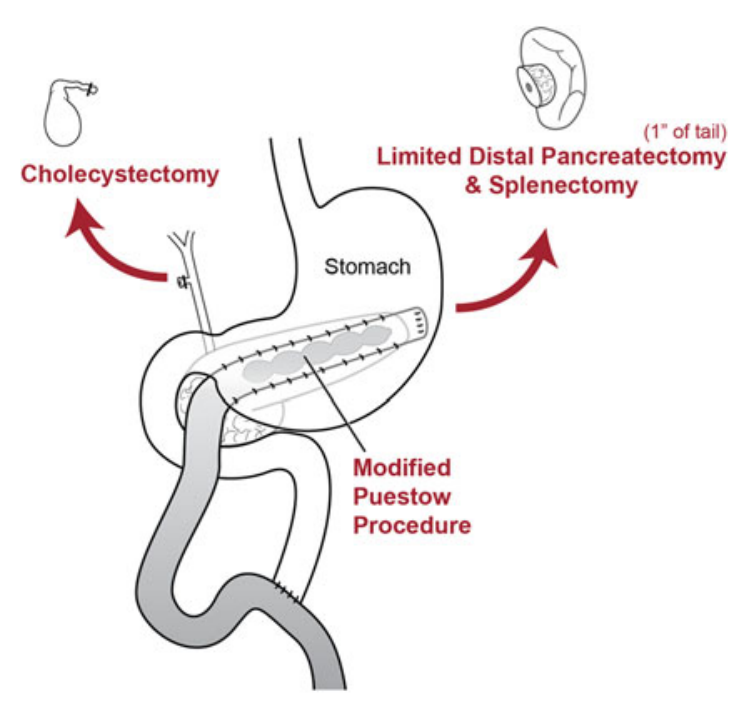

First Procedure (1992)

FIG. 1. Diagram of the initial modified Puestow procedure.

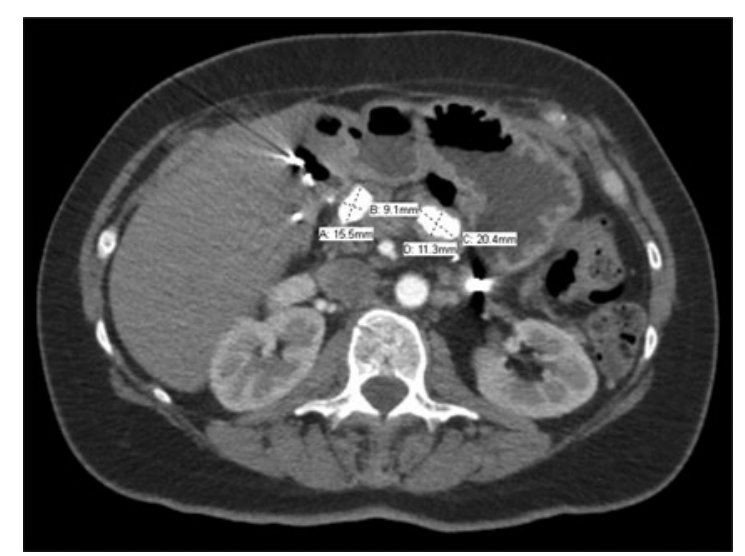

FIG. 2. Computed tomography image showing two substantial calcifications in the pancreatic remnant and their measured size. Note the absence of the spleen in the left upper quadrant.

nerve block was performed by injecting $20 \mathrm{~mL}$ of $50 \%$ ethanol solution on either side of the aorta at the level of the celiac axis. The pathology on the resection specimen demonstrated chronic pancreatitis with parenchymal calcifications and duct ectasia consistent with PRSS1-related HP. No cancer was seen. The patient recovered well, was discharged on postoperative day 5 , and was seen in follow-up fully recovered.

\section{Discussion}

HP is an important cause of childhood pancreatitis, with a median age of symptom onset reported as ranging from 5 to 10 years and with a median age of diagnosis reported as ranging from 6 to 19 years. ${ }^{1,2} \mathrm{HP}$ is most easily distinguished from non-HP in two ways: HP presents earlier in life, commonly in the first or early second decade of life, and it carries an increased risk of pancreatic adenocarcinoma (standardized incidence ratio 87). ${ }^{1,3,9}$ Despite an earlier age of onset, the hereditary nature of HP often goes undiagnosed. Nearly $50 \%$ and $75 \%$ of children diagnosed with acute recurrent pancreatitis and chronic pancreatitis, respectively, have an underlying genetic predisposition to their disease. ${ }^{4}$ The prevalence of HP has been estimated to be $0.3 / 100,000$ people in France. ${ }^{1}$ Numerous gene mutations have been identified as causative or disease modifying for HP, the four most common being PRSS1, SPINK1, CFTR, and CTRC ${ }^{3-5}$ (Table 1). As already mentioned, the disease course of HP typically mirrors that of recurrent acute pancreatitis often culminating in a clinical picture of chronic pancreatitis. It should, 

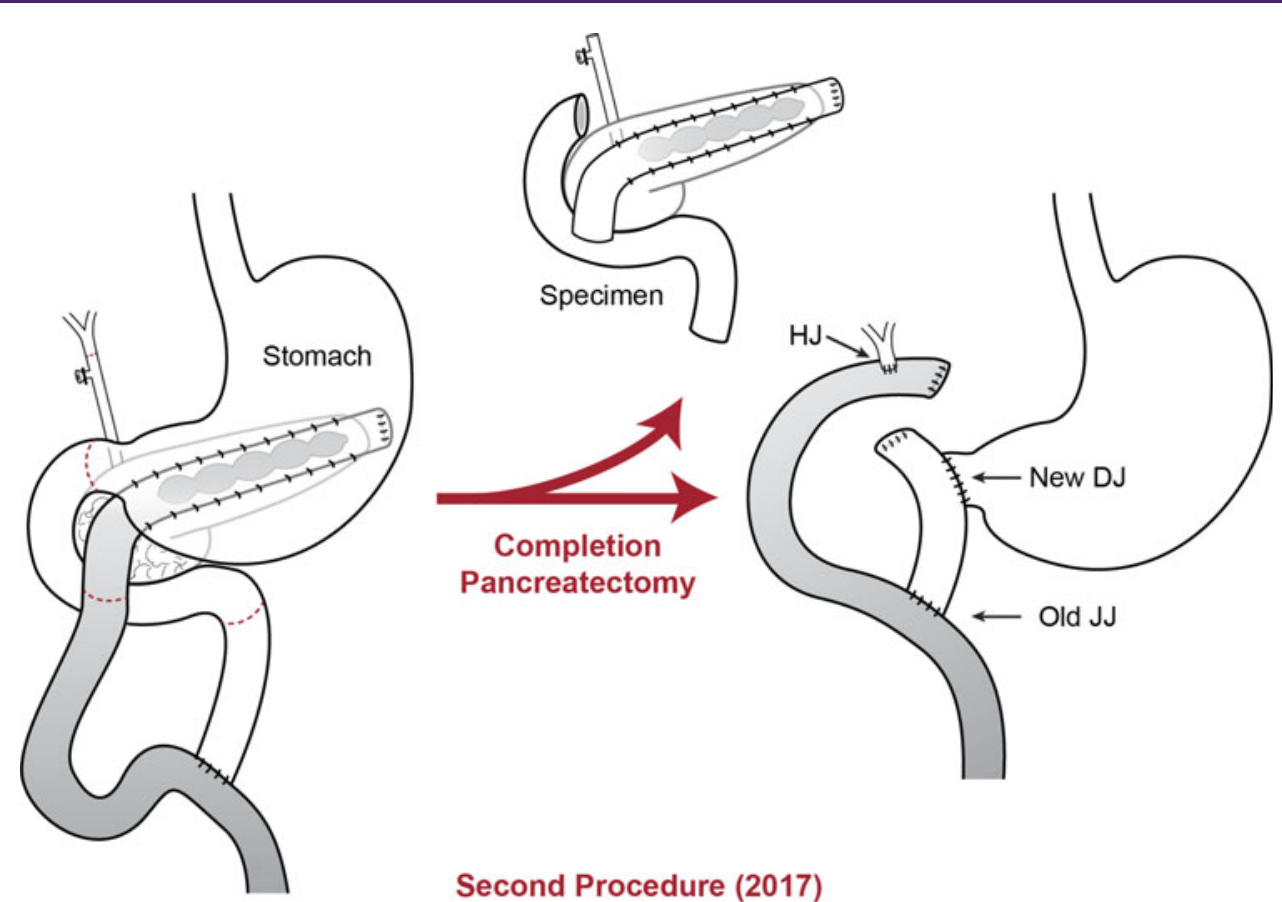

Second Procedure (2017)

FIG. 3. Diagram of the completion pancreaticoduodenectomy procedure. DJ, duodenojejunostomy; $\mathrm{HJ}$, hepaticojejunostomy, JJ, jejunojejunostomy.

Table 1. Common Gene Mutations in Hereditary Pancreatitis

\begin{tabular}{lllll}
\hline Gene & Cr & \multicolumn{1}{c}{ Inheritance } & Disease relationship & Mutation \\
\hline PRSS1 & $7 q 34$ & Autosomal dominant & Disease causing & Gain of function \\
SPINK1 & $5 q 32$ & Complex (most commonly autosomal recessive) & Usually disease modifying & Loss of function \\
CFTR & $7 q 31.2$ & Autosomal recessive & Disease modifying & Loss of function \\
CTRC & $1 \mathrm{p} 36.21$ & Unreported & Disease modifying & Loss of function \\
\hline
\end{tabular}

however, be noted that some patients first present with chronic pancreatitis devoid of previous acute pancreatitis symptoms. ${ }^{10}$ Features of HP typically include classic pancreatic pain, nausea, and vomiting. In addition, pancreatic calcifications, parenchymal pseudocysts, and ductal abnormalities may be seen, often culminating in cholestasis, diabetes mellitus, and steatorrhea. ${ }^{1}$ Repeated episodes of pancreatitis often result in frequent interactions between medical providers and patients with HP. ${ }^{10}$ Treatment regimes include mitigation of risk factors (e.g., smoking and alcohol use), medical management through pancreatic enzyme supplementation and pain control, endoscopic pancreatic duct decompression, and surgical therapy. Treatment considerations must include the increased relative and absolute risk of pancreatic adenocarcinoma in patients with HP where the median age of diagnosis is 55 years. ${ }^{1,9}$

\section{Conclusion}

A completion pancreatectomy in patients with HP can be performed after previous pancreatic surgical intervention to treat disease manifestations and as a prophylaxis against the increased risk of pancreatic adenocarcinoma.

\section{Acknowledgments}

The authors thank Jennifer Brumbaugh for the preparation of Figure 1 and Figure 3.

\section{Author Disclosure Statement}

No competing financial interests exist for authors J.R.N., A.G.S., or C.J.Y.

\section{References}

1. Rebours V, Boutron-Ruault MC, Schnee M, et al. The natural history of hereditary pancreatitis: a national series. Gut. 2009;58:

97-103. 
2. Sultan M, Werlin S, Venkatasubramani N. Genetic prevalence and characteristics in children with recurrent pancreatitis. J Pediatr Gastroenterol Nutr. 2012;54:645-650.

3. Whitcomb DC. Genetic predispositions to acute and chronic pancreatitis. Med Clin North Am. 2000;84:531-547, vii.

4. Kumar S, Ooi CY, Werlin S, et al. Risk factors associated with pediatric acute recurrent and chronic pancreatitis: lessons from INSPPIRE. JAMA Pediatr. 2016;170:562-569.

5. Vue PM, McFann K, Narkewicz MR. Genetic mutations in pediatric pancreatitis. Pancreas. 2016;45:992-996.

6. Whitcomb DC, Gorry MC, Preston RA, et al. Hereditary pancreatitis is caused by a mutation in the cationic trypsinogen gene. Nat Genet. 1996;14:141-145.

7. Howes N, Lerch MM, Greenhalf W, et al. Clinical and genetic characteristics of hereditary pancreatitis in Europe. Clin Gastroenterol Hepatol. 2004; 2:252-261.

8. Canto Ml, Harinck F, Hruban RH, et al. International Cancer of the Pancreas Screening (CAPS) Consortium summit on the management of patients with increased risk for familial pancreatic cancer. Gut. 2013;62:339-347.

9. Rebours V, Boutron-Ruault MC, Schnee M, et al. Risk of pancreatic adenocarcinoma in patients with hereditary pancreatitis: a national exhaustive series. Am J Gastroenterol. 2008;103:111-119.
10. Patel MR, Eppolito AL, Willingham FF. Hereditary pancreatitis for the endoscopist. Therap Adv Gastroenterol. 2013;6:169-179.

Cite this article as: Nellen JR, Strickland AG, Yeo CJ (2018)

Completion pancreaticoduodenectomy for hereditary pancreatitis after prior Puestow procedure: a case report, Journal of Pancreatic Cancer 4:1, 60-63, DOI: 10.1089/pancan.2018.0012.

\section{Abbreviations Used}

$C F T R=$ cystic fibrosis transmembrane conductance receptor $C T R C=$ chymotrypsin $\mathrm{C}$

$\mathrm{HP}=$ hereditary pancreatitis

PRSS1 $=$ protease serine 1

$S P I N K 1=$ serine protease inhibitor Kazal type 1

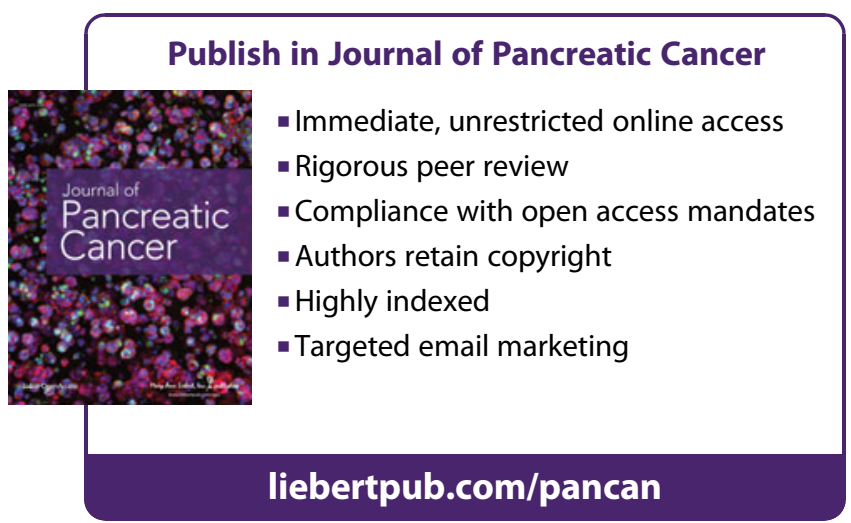

